\title{
Immunolocalization of dynein, dynactin, and kinesin in the cerebral tissue as a possible supplemental diagnostic tool for traumatic brain injury in postmortem examination
}

\author{
Mieszko Olczak ${ }^{1}$, Łukasz Poniatowski ${ }^{2,3}$, Magdalena Kwiatkowska ${ }^{1}$, Dorota Samojłowicz ${ }^{1}$, Sylwia Tarka ${ }^{1,4}$, \\ Teresa Wierzba-Bobrowicz ${ }^{4}$ \\ ${ }^{1}$ Department of Forensic Medicine, Medical University of Warsaw, Warsaw, ${ }^{2}$ Department of Experimental and Clinical Pharmacology, \\ Centre for Preclinical Research and Technology (CePT), Medical University of Warsaw, Warsaw, ${ }^{3}$ Department of Neurosurgery, \\ Maria Skłodowska-Curie Memorial Cancer Center and Institute of Oncology, Warsaw, ${ }^{4}$ Department of Neuropathology, Institute \\ of Psychiatry and Neurology, Warsaw, Poland
}

\begin{abstract}
Traumatic brain injury (TBI) is characterized by various micro- and macrostructural neuropathological changes which can be identified in the light microscope examination. The most common pathophenotype of TBI visualized in postmortem neuropathological assessment includes neuron injury with involvement of all of its structural regions followed by its progressive degeneration defined as traumatic axonal injury (TAI). This is directly related with disruption of the axolemmal cytoskeletal network architecture resulting in breakdown, dissolution and accumulation of a number of neuronal proteins. Regarding the availability and progress in the development of specific antibodies against neuronal proteins, their usage is restricted due to low specificity for injured axons in the pathomechanism of TBI followed by TAI. Taking this into account with relation to expanding the role of axonal cytoskeleton and its based biomarkers we have presented a study documenting neuropathological features concerning the expression of dynein (DNAH9), dynactin (DCTN1) and kinesin (KIF5B) in the brain specimens obtained during forensic autopsies from TBI victims. The study was carried out using cases $(n=21)$ of severe head injury suspected to be the cause of death and control cases $(n=17)$ of sudden death in the mechanism of cardiopulmonary failure along with a positive control case which died after suicidal gunshot injury. In our study, we documented that DNAH9, DCTN1, and KIF5B staining should be considered as a supplemental diagnostic tool for TBI in postmortem neuropathological examination and forensic autopsy. This additional motor protein immunohistochemical staining procedure could be useful in the evaluation of lesions that may remain undiagnosed during a routine examination and aid in more accurate identification of TBI followed by TAI.
\end{abstract}

Key words: dynein, dynactin, kinesin, axon pathology, traumatic brain injury.

\section{Introduction}

Traumatic brain injury (TBI) is recognized as one of the most devastating clinico-pathological states [9]. Despite continuous advances in the neuroin- tensive care with the implementation of a targeted combined medical-surgical approach, TBI still represents the leading cause of death and disability worldwide [54,62]. Regarding epidemiological data, it was estimated that annually TBI is associated with

Communicating author

Mieszko Olczak, MD, PhD, Department of Forensic Medicine, Medical University of Warsaw, 1 Oczki St., 02-007 Warsaw, Poland, e-mail: mieszkothe1st@hotmail.com 
10 million deaths and hospital admissions which results collectively in the fact that globally 57 million living people have been hospitalized with single or more episodes of TBI [40]. Therefore these short statistical data mentioned represent that each year a large part of the population across the world, both from the civilian and military sector, is affected by $\mathrm{TBI}$ and its long-lasting consequences. The natural course of the heterogeneous condition such as TBI is related to two subsequent and not clearly demarcated pathophysiological events such as primary and secondary (delayed) injury where practically all areas, levels, and compartments of the brain could be affected [73]. Primary injury is defined as the direct effect of a transiently applied external physical force, whose nature and other combined attributes affect the severity of the injury [46]. Applied initial primary injury can be a result of direct/indirect blunt external mechanical force, rapid acceleration/ deceleration, blast arising from shockwave exposure and ballistic penetration by projectile [18]. The variety of changes occurring at the moment of trauma include mechanical damage of cranium, meninges and soft tissues associated with collective injury of grey and white matter occurring with rapid proteolysis of the axoplasm, what is defined as primary axotomy [7]. Primary injury-associated events are also influenced by the development of intra- and extra-axial space-occupying mass lesions such as hematomas and oedematous changes in neural tissue accompanied by alternations in hydrodynamics of the cerebrospinal fluid (CSF) [30]. On the cellular and microstructural level, screening of the primary injury effects includes the immediate death of neurons, glia and therefore endothelial cells resulting in the swift release of neurotransmitters along with disruption of microvascular regulation and permeability ongoing with a dysfunction of neurovascular units and junction proteins forming the blood-brain barrier (BBB) $[14,27]$. Secondary injury comprises nonlinear self-propagating events representing the continuation of the processes initiated by primary injury ongoing from minutes to years [38]. These synergistic neurometabolic and neurochemical mechanisms include but are not limited to further alternations in vascular regulation, evoke oxidative stress (OxS) accompanied with free-radical production, peroxidation (PX) of macromolecules, glutamate (Glu) resilient accumulation/excitotoxicity and neuroinflammation $[8,21]$. Among both phases of
$T B I$, one of the integral pathophenotypes includes disruption of integral and functional maintenance of neuronal cell membrane and axolemmal cytoskeletal network architecture which are together defined as a traumatic axonal injury (TAI) [11]. An additive mechanism is associated with a complex failure of axonal mitochondria metabolism due to the impairment of adenosine-5'-triphosphate (ATP) production along with alternations in its transmembrane potential $(\Delta \Psi)[68]$. Ongoing and exacerbated disequilibrium of axolemma from stretching caused an uncontrolled influx of sodium $\left(\mathrm{Na}^{+}\right)$and calcium $\left(\mathrm{Ca}^{2+}\right)$ ions from extracellular space accompanied by an intra-axonal release of the latter ones from mitochondria and axoplasmic reticulum [70]. Initiated $\mathrm{Ca}^{2+}$-dependent influx waves cascade causes consequent proteolysis of the key axonal cytoskeleton components including neurofilaments, microtubules and subaxolemmal actin/spectrin complex [58]. This process is mediated through $\mathrm{Ca}^{2+}$-sensitive enzymes such as the number of proteases, calpains, phosphatases and phospholipases $[43,44]$. These reviewed ultrastructural and neuropathological events during post-acute and chronic phases of TBI associated with a progressive disruption in axonal transport, interruption of terminal axonal connections, demyelination and neuronal loss are also defined as secondary axotomy which is followed by next-step Wallerian degeneration [6]. The important role of axonal cytoskeleton and its based biomarkers in TBI models has increased in recent years [32]. Although the wide availability of antibody panels allow us to perform visualization of axonal disruption in a neuropathological examination, their usage is restricted due to the low specificity concerning damaging circumstances. However, despite these findings, along with ongoing development of immunocytochemical labelling techniques, there is still a need to attempt to identify novel supplemental markers and their compilation platforms for injured nerve fibers as a result of TBI. Functions and mechanics of the axoplasmic cytoskeleton network are associated with the three large families of ATP-fuelled motor proteins such as dynein, dynactin and kinesin [45]. Consecutively, these multisubunit complexes are involved in a variety of essential functions in eukaryotic cells such as bidirectional transport of biological cargoes and vesicles that include, but are not limited to proteins, macromolecules, endosomes, mitochondria and other organelles to target regions along with regulation 
of mitosis, meiosis, autophagy and maintenance of the endoplasmic reticulum and Golgi apparatus $[20,48]$. According to the topological superstructure, dynein constitutes a complicated multiprotein complex ( 1.5 MDa) built around a pair of identical heavy chains that are connected with two hexameric rings belonging to ATPases associated with diverse activities (AAA+) superfamily and dimeric subunits such as intermediate chains, light-intermediate chains and light chains (TCTEX, LC8 and Roadblock) [52]. Dynactin, an accessory adaptor protein $(\sim 1.2$ $\mathrm{MDa}$ ) is composed of 11 different subunits and interacts with dynein via its $\mathrm{N}$-terminal intermediate chain $[39,67]$. This assembled heterooligomeric complex translocates towards the minus end (retrograde) and applies a pulling force on the microtubule network through repeating mechanochemical cycle obtaining energy from ATP hydrolysis [13]. Accordingly, kinesin holoenzyme frequently displaces along microtubules in the opposite plus end (anterograde) direction, acting as a conventional cross-linked heterotetramer of two light chains $(\sim 65 \mathrm{kDa})$ and two heavy chains $(\sim 120 \mathrm{kDa})$ containing the $\mathrm{N}$-terminal motor domain [33]. Although the role of microtubules in cytoskeletal derangement in case of TBI has been widely characterized, less is known about the role of its associated motor proteins. Reflecting on the physiological role, distribution, and features of motor proteins in the highly polarized and coordinated cytoskeleton system, in neuronal cells we hypothesize that they could serve as a potential supplemental diagnostic marker indicating the axonal disruption in case of TBI for neuropathological and forensic purposes. According to these facts, we conducted a study to attempt to preliminarily evaluate and qualitatively describe the expression of dynein, dynactin, and kinesin by performing immunohistochemical staining within the brain tissue of the analyzed population in postmortem examination. To our best knowledge, the currently available literature does not contain similar published studies to the one presented in this manuscript, that is, pertaining to the usage of motor protein staging in the neuropathological examination of TBI.

\section{Material and methods Autopsy cases}

The study was carried out using 21 cases $(n=21)$ of severe head injury suspected to be the cause of death such as contusions, abrasions and lacerations visible on the skin of the head comprising deaths not preceded by hospitalization and cardiopulmonary resuscitation (CPR) along with 17 cases $(n=17)$ of sudden death in the mechanism of cardiopulmonary failure resulting from cardiac arrest due to acute myocardial failure (no injuries of the head) without cardiopulmonary resuscitation (CPR). The positive control was included and defined using one 52-yearold male decedent $(n=1)$ which died instantly after direct projectile penetration of the head and brain resulting from a suicidal gunshot injury and CPR was not performed. All cases to be evaluated were provided by forensic pathologists from the Department of Forensic Medicine at the Medical University of Warsaw. Additional information about the circumstances of death was collected from the case files. The following study is based on case groups as presented in our previous publications [50,51]. The average age of the deceased was $53.7 \pm 3.9$ in the study group and $55.8 \pm 3.0$ in the control group. Groups did not differ statistically $(p>0.05)$ with respect to age.

\section{Tissue dissection and preparation}

At the time of forensic autopsy carried out within 24-48 hours after death (cases with visible signs of putrefaction were excluded from the study), brain specimens comprising the frontal lobe associated with cingulate cortex and corpus callosum were obtained and fixed in $10 \%$ buffered formalin $\left(\mathrm{CH}_{2} \mathrm{O}\right)$, then embedded in paraffin. No signs of neurodegeneration were observed during neuropathological examination of brain sections. Also, both medical records and family testimony did not report neurodegenerative brain disease history in any of the patients. Control group cases revealed no morphological signs of a brain injury either on the macroscopic or microscopic level. The study group revealed blunt injuries to the head (contusions, abrasions, lacerations or hemorrhages in the subcutaneous tissue of the head) on the postmortem examination. The injuries were resulted in some cases in macro- and microscopic signs of a brain injury. Part of the study group ( $52 \%$ of cases, $n=11$ ) were primary lethal (subdural hemorrhage, brain contusion or intraventricular bleeding). Another part of the study group ( $48 \%$ of cases, $n=10$ ) revealed minor posttraumatic neuropathological findings such as local subarachnoid hemorrhage (SAH) that could not be precisely assumed to be the cause of death. In these cases, the 
cause of death was linked to multiple trauma (injuries to the head accompanied by lethal injuries to the trunk). Sample brain lesions seen in hematoxylin and eosin (H\&E) and other various immunohistochemical staining of the groups were presented in our previous works $[50,51]$. Dissected intact specimens were stained immunohistochemically with the dynein heavy chain (DNAH9) antibody (NBP1-90492; Novus Biologicals, Littleton, CO, USA) in 1 : 100 dilution, dynactin subunit 1 (DCTN1) antibody (AB6048; Merck Millipore, Burlington, MA, USA) in 1 : 100 dilution and kinesin family member 5B (KIF5B) antibody (21632-1-AP; Proteintech, Chicago, IL, USA) in 1 : 100 dilution according to the protocols supplied by the manufacturers. Positive control (gunshot injury) specimens were additionally stained with $\mathrm{H} \& \mathrm{E}$ along with the amyloid precursor protein (APP) antibody (NB110-55461; Novus Biologicals, Littleton, CO, USA) in $1: 250$ dilution according to the manufacturer's protocols. The microphotographs of the brain sections were taken with the Olympus BX51 microscope (Olympus Optical, Tokyo, Japan) equipped with Camedia C-3040 Zoom digital camera (Olympus Optical, Tokyo, Japan) connected with computerized data acquisition and image analysis system, Olympus DP-Soft version 3.2 software (Olympus Soft Imaging Solutions, Tokyo, Japan).

\section{Results}

\section{Dynein}

In the neocortex, the control group presented a positive DNAH9 expression in cytoplasm corti- cal neurons and axons distinctly increased in the vicinity/area of the nucleus with no reaction in its central area. The study group revealed distinctly diminished homogeneous DNAH9 expression in the cytoplasm of cortical neurons and axons. We did not observe significant changes among corpus callosum in DNAH9 expression between the study and control group. Furthermore, the intensity of observed changes tends to increase with the severity of the head injury sustained. A positive control (gunshot injury) case presented positive DNAH9 expression in the neuropil (outside neural cells) seen as numerous lumps. Sample microphotographs showing DNAH9 expression are presented in Figure 1.

\section{Dynactin}

In the neocortex, the control group presented a positive DCTN1 expression (positive reaction with clusters/graininess formation) in the cytoplasm of cortical neurons simultaneously with a positive expression in axons. The study group revealed distinctly diminished homogeneous DCTN1 expression in the cytoplasm of cortical neurons with almost no expression in axons with a positive reaction also seen in the form of lumps located in the neuropil. In the corpus callosum, the control group presented a positive and steady DCTN1 expression along axons. In the study group, a positive increased DCTN1 expression was segmentally seen in axon fragments. Furthermore, a positive reaction was seen in the form of lumps located between the axons. The number and
A

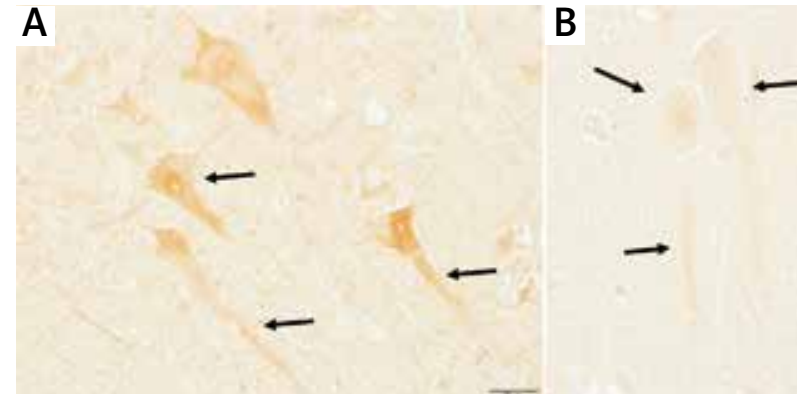

C

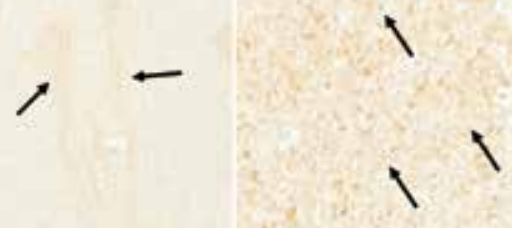

Fig. 1. Representative microphotographs showing DNAH9 expression in examined brain sections. A) Control group, frontal cortex at $\times 400$ magnification; positive DNAH9 reaction in the cytoplasm of cortical neurons of the $5^{\text {th }}$ layer and its axons distinctly increased in the area of the nucleus with no reaction in its central area (black arrows). B) Study group (primary lethal), frontal cortex at $\times 400$ magnification; distinctly diminished homogeneous DNAH9 expression in the cytoplasm of cortical neurons of the $5^{\text {th }}$ layer and its axons (black arrows). C) Positive control (gunshot injury) case, white matter in the vicinity of the bullet channel at $\times 400$ magnification; positive disseminated DNAH9 reaction in the neuropil (outside neural cells) seen as numerous lumps in the vicinity of brain gunshot lesions (black arrows). 

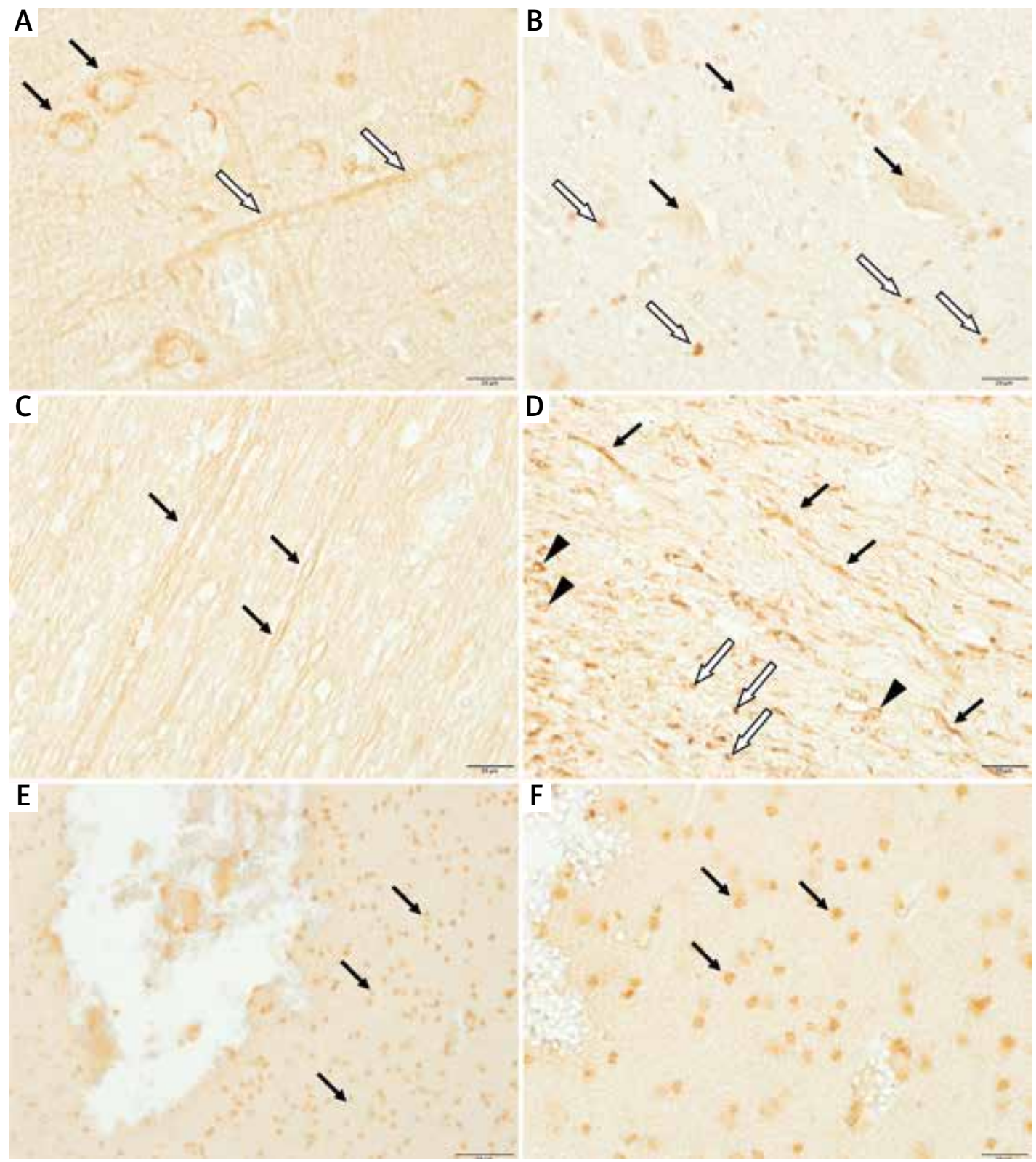

Fig. 2. Representative microphotographs showing DCTN1 expression in examined brain sections. A) Control group, frontal cortex at $\times 400$ magnification; positive DCTN1 reaction (with clusters/graininess formation) in the cytoplasm of cortical neurons of the $5^{\text {th }}$ layer (black arrows) simultaneously with positive expression in axons (white arrows). B) Study group (primary lethal), frontal cortex at $\times 400$ magnification; distinctly diminished homogeneous DCTN1 expression in the cytoplasm of cortical neurons of the $5^{\text {th }}$ layer (black arrows) with almost no expression in axons, the positive reaction also seen in the form of lumps located in the neuropil (white arrows). C) Control group, corpus callosum at $\times 400$ magnification; positive and steady DCTN1 reaction along axons (black arrows). D) Study group (primary lethal), corpus callosum at $\times 400$ magnification; positive increased DCTN1 reaction segmentally seen in axon fragments (black arrows), the positive reaction is also seen in the form of lumps located between the axons (white arrows) and in glial-like cells (black triangles). E) Positive control (gunshot injury) case, white matter in the vicinity of the bullet channel at $\times 200$ magnification; positive disseminated DCTN1 reaction in the vicinity of brain gunshot lesions (black arrows). F) Positive control (gunshot injury) case, white matter in the vicinity of the bullet channel at $\times 400$ magnification; positive disseminated DCTN1 reaction in glial-like cells in the vicinity of brain gunshot lesions (black arrows). 
severity of observed changes tends to increase with the severity of the head injury sustained. The positive control (gunshot injury) case presented a clear DCTN1 expression in neuroglial cells in the vicinity of the bullet channel, which have been identified basing on cell morphology seen on H\&E stainings of the next sections. Sample microphotographs showing DCTN1 expression are presented in Figure 2.

\section{Kinesin}

In corpus callosum, the control group presented a positive KIF5B expression in axons and oligodendroglia which have been identified basing on cell morphology seen on H\&E stainings of the next sections. The study group presented an enhanced KIF5B expression segmentally seen in axon fragments with a diminished expression in oligodendroglia. As in the two previous stainings, the intensity of observed changes tends to increase with the severity of the head injury sustained. Some of the study group cases presented cells with a positive KIF5B expression in the vicinity of blood vessels in white matter of frontal lobes (simultaneously red blood cells in the lumen of vessels presented no KIF5B expression), other cases presented simultaneously cells with a positive KIF5B expression in the lumen of cortical blood vessels in the same area. We did not observe significant changes among cortical neurons in KIF5B expression between the control and study group. The positive control (gunshot injury) case presented positive KIF5B expression cells in the vicinity and in the lumen of blood vessels in white matter, in the vicinity of the bullet channel, as well as in neuroglia and neuropils (outside neural cells) seen as numerous lumps where neuroglia were identified basing on cell morphology seen on $H \& E$ stainings of the next sections. Sample microphotographs showing KIF5B expression are presented in Figure 3.

\section{Positive control (H\&E and APP)}

The positive control (gunshot injury) case presented numerous hemorrhagic foci in the vicinity of the bullet channel with a visible reaction of neuroglia and single macrophages (increased numbers) where neuroglia and macrophages were identified basing on cell morphology seen on $H \& E$ stainings of the next sections. At the same time, there were no retraction bulbs seen in APP staining. Sample microphotographs showing H\&E and APP stainings in the positive control (gunshot injury) case are presented in Figure 4.

\section{Discussion}

Pathophysiology of TBI establishes a number of heterogeneous micro- and macrostructural neuropathological changes which can be observed and characterized in the light microscope examination. The nature of this circumstance directly results from complex brain cytoarchitecture which includes a large number of interacting cell populations and regions, each with its dedicated and distinctive function $[4,5]$. These observations are mainly related to neurons which consist of morphologically complex and highly specialized asymmetric polarized cells responsible for generation, conduct, receipt and transmission of neurochemical impulses [35]. Among them, glial cells such as astrocytes, oligodendrocytes, and microglia also exert their dedicated supportive properties regulating and maintaining the microenvironment of cerebral tissue [36]. At the moment of instantaneous application of biomechanical force and its mechanotransduction, the entire integrated cell populations mentioned and scales composing brain parenchyma are affected [31]. However, the most important and common consequence of TBI observed in postmortem neuropathological assessment is associated with a collective neuron injury, covering all of its parts such as the body, axon, and dendrites followed by its ongoing instability and progressive degeneration referred to as TAI [6]. This is directly related with alternations concerning components of the neuronal cytoskeleton such as breakdown, dissolution, and accumulation of microtubules, neurofilaments and subaxolemmal actin/spectrin complexes situated along the length of the axon [56]. Certain morphological features in predefined contusional cerebral regions of white matter following TBI are directly reflected by an interspersed occurrence of axonal swelling occurring within hours after the trauma due to the accumulation of transported cellular cargoes $[15,60]$. The periodic organization of swellings interconnected by a contiguous axon along its length at the location of the injury is more recently referred to as axonal varicosities $[63,64]$. Another phenotype morphological manifestation of axonal disruption certainly representing its complete disconnection is one large single insertion known as retraction bulb which is 
A

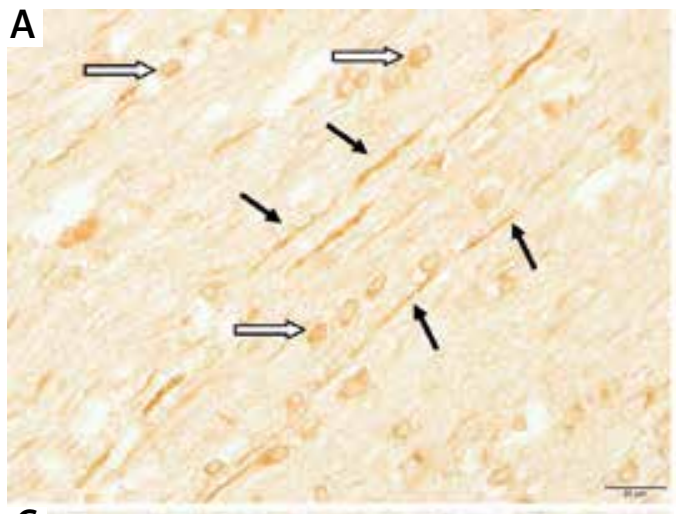

C
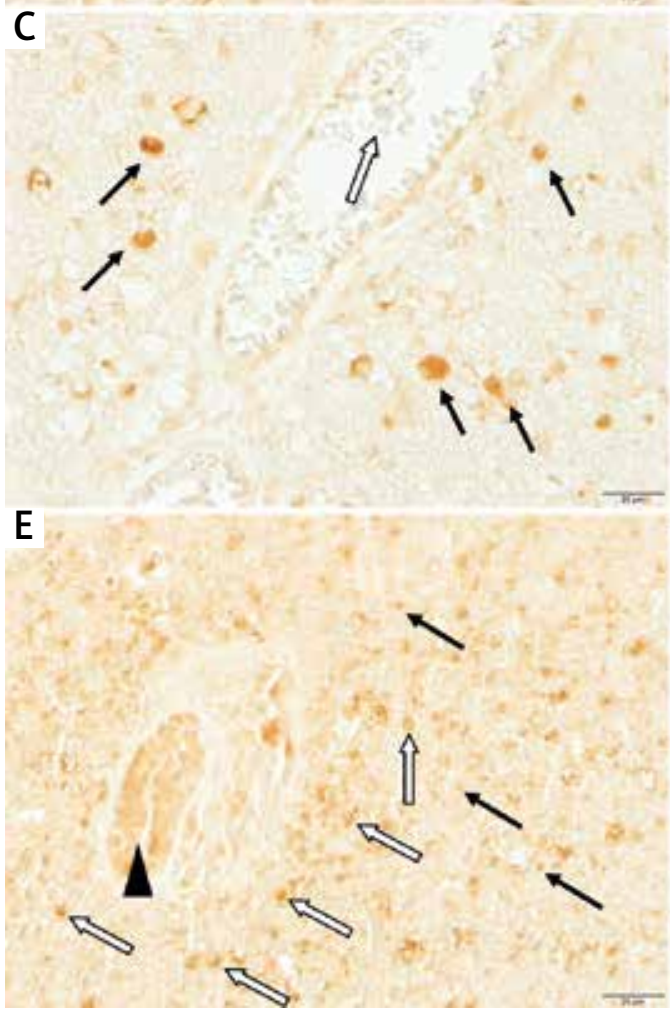

B

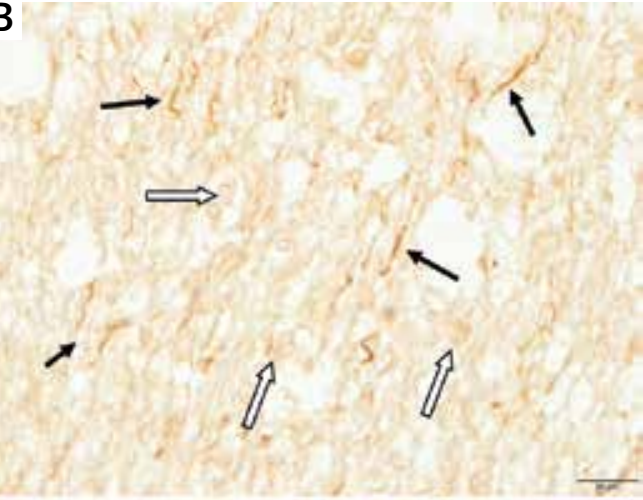

D
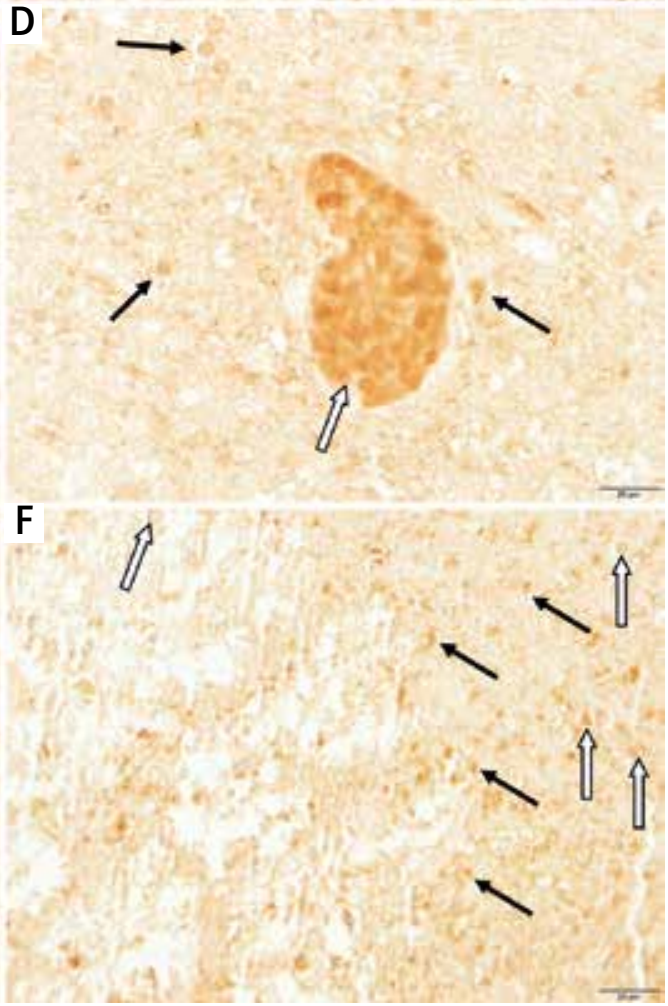

Fig. 3. Representative microphotographs showing KIF5B expression in examined brain sections. A) Control group, corpus callosum at $\times 400$ magnification; positive and steady KIF5B reaction along axons (black arrows), the positive KIF5B reaction in oligodendroglia-like cells (white arrows). B) Study group (primary lethal), corpus callosum at $\times 400$ magnification; positive increased KIF5B reaction segmentally seen in axon fragments (black arrows), diminished KIF5B reaction in oligodendroglia-like cells (white arrows). C) Study group (primary lethal), white matter of the frontal cortex at $\times 400$ magnification; KIF5B positive cells most likely macrophages/phagocytic microglia in the vicinity of a blood vessel (black arrows), red blood cells in the lumen of a blood vessel with no KIF5B reaction (white arrow). D) Study group (primary lethal), white matter of the frontal cortex at $\times 400$ magnification; KIF5B positive cells most likely macrophages/phagocytic microglia in the vicinity of a blood vessel (black arrows), KIF5B positive cells in the lumen of a blood vessel (white arrow). E) Positive control (gunshot injury) case, white matter in the vicinity of bullet channel at $\times 400$ magnification; positive disseminated KIF5B reaction in neuropils (outside neural cells) seen as numerous lumps (black arrows), KIF5B positive cells most likely macrophages/phagocytic microglia in the vicinity of a blood vessel (white arrows), KIF5B positive cells in the lumen of a blood vessel (black triangle). F) Positive control (gunshot injury) case, white matter in the vicinity of the bullet channel at $\times 400$ magnification; positive disseminated KIF5B reaction in neuropils (outside neural cells) seen as numerous lumps in the vicinity of brain gunshot lesions (black arrows), positive KIF5B reaction in neuroglial-like cells (white arrows). 

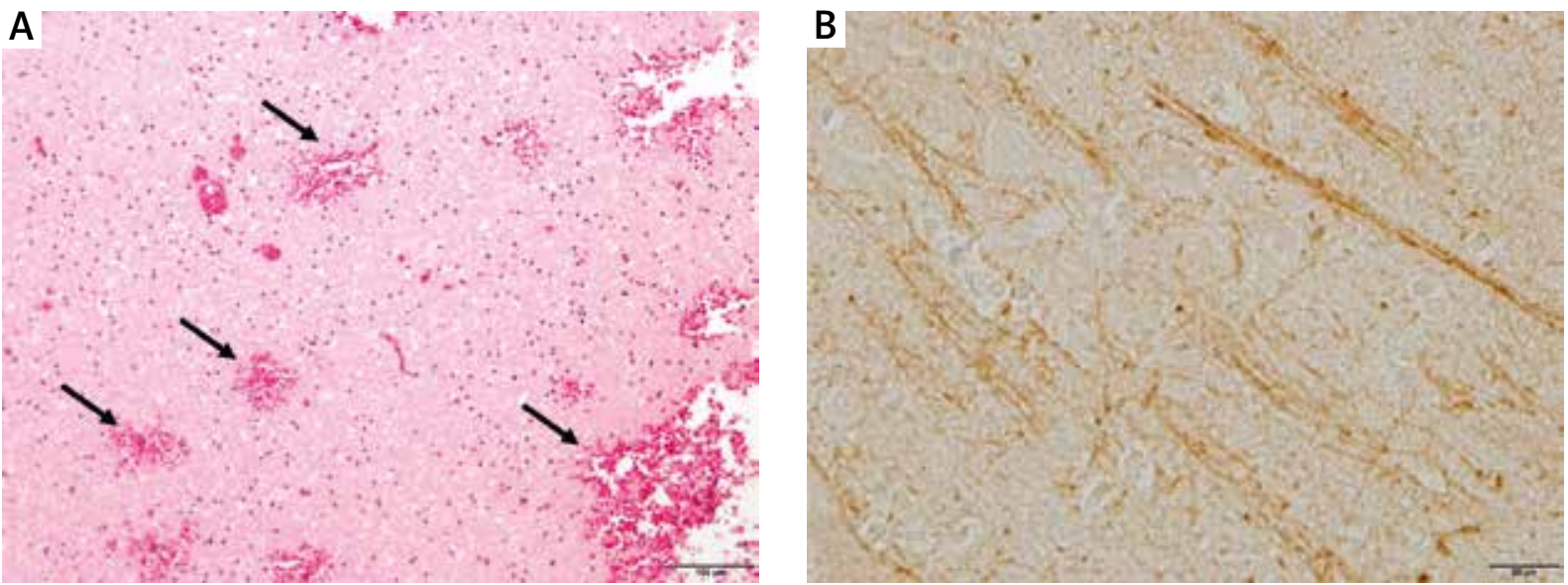

Fig. 4. Representative microphotographs showing H\&E and APP expression in examined positive control (gunshot injury) case brain sections. A) Positive control (gunshot injury) case in H\&E staining, white matter in the vicinity of the bullet channel at $\times 100$ magnification; gunshot lesions along with hemorrhagic foci (black arrows). B) Positive control (gunshot injury) case in APP staining, white matter in the vicinity of the bullet channel at $\times 400$ magnification; no visible retraction bulbs.

found in a short period of time after TBI [29]. This image is usually hallmarked by the presence of microglial scars, disseminated gliosis, microhemorrhages and long tract degeneration [16]. Taking into consideration that TBI has a disseminated and multifocal nature, the visualization and diagnosis of this condition premortem could be indistinguishable or undetectable to current non-invasive routine radiological and nuclear medicine techniques where the postmortem neuropathological examination mainly provides its definitive diagnosis $[28,41]$. Although, in particular novel, advanced neuroimaging techniques such as diffusion tensor imaging (DTI) may be promising in the evaluation of white matter tracts integrity in vivo through estimation of the diffusion anisotropy of water molecules [71]. Across various histopathological approaches applied to date for tinctorial stainings visualization of TAI and its abnormal axonal phenotypes, one should list H\&E, silver impregnation including Palmgren method, sole 3,3',5,5'-tetramethylbenzidine (TMB) staining and conventional myelin stainings $[2,19,49,65]$. Currently used immunohistochemical stainings utilizing targeted antibodies remain the gold standard in the diagnosis of TAI and its distribution for both experimental and clinical purposes in the field of neuropathology and forensic medicine [60]. Accordingly, from multiple candidates staining with APP antibodies attracted significant attention due to its sensitivity and robust capacity to identify injured axons in individuals within $\sim 30 \mathrm{~min}$ after TBI [26,34]. Additional immunohistochemical staining used for examination of proteins whose exacerbated expression is detected in the course of TBI includes, but is not limited to presenilin-1 (PS-1) antibodies, beta ( $\beta$ ) amyloid cleaving enzyme (BACE) antibodies, alpha ( $\alpha$ )-synuclein (ASN) antibodies, ubiquitin (Ub) antibodies, cytochrome c (CYCS) antibodies and caspase-3 (CASP3) antibodies along with a number of axonal cytoskeleton labelled antibodies including microtubule-associated protein tau (MAPT), calpain-mediated spectrin proteolysis (CMSP), $68 \mathrm{kDa}$ neurofilament (NF), phosphorylated $200 \mathrm{kDa}$ NF (SMI-31) and non-phosphorylated $150 \mathrm{kDa} N F$ (SMI-32) $[10,12,55,66,72]$. Regarding the ongoing progress in characterization and utilization of new antibodies and their combinations against neuronal proteins, they still do not provide exclusive specific labeling for disrupted axons in the pathomechanism of TBI followed by TAI [37]. In this case, accumulated proteins such as APP are also present during a hypoxic/ ischemic or hemorrhagic insult along with neurodegenerative diseases [47]. Another limiting concern besides damaging circumstances specificity is associated with over- and under-interpretation in the evaluation of the scale and time evolution of axonal injury which is critical in the assessment of TAI $[37,57]$. In this regard and in relation to the growing role of the axonal cytoskeleton and its based biomarkers in TBI studies, we have presented a study 
documenting various neuropathological features concerning the expression of dynein, dynactin, and kinesin in the obtained cerebral slices from TBI victims and their comparable control group cases. In general, our study group showed a diminished homogeneous expression in neurons and axons concerning DNAH9 and DCTN1 with no significant changes in KIF5B expression among cortical neurons of the $5^{\text {th }}$ layer in neocortex. Additional finding concerning DNAH9 was associated with its overexpression around the nucleus with no reaction in its central area, what is consistent with centrosome localization and functioning [53]. In relation to findings observed within the corpus callosum, the DNAH9 revealed no significant changes in expression between the study and control group whereas DCTN1 and KIF5B have shown a positive increased expression localized segmentally in axon fragments. This is corresponding with classic findings regarding partial interruption segmentally interconnected by a contiguous axon fragment along its length at the injured site [25]. Due to the short period of time between TBI and death, we suppose that axon disruption consists of a direct effect of injury rather than potential hypoxic/ischemic effects which could also develop as the effect of prolonged agony phase or CPR which was not performed in any of our groups. In our previous study, using a series of antibodies against APP, NF, myelin basic protein (MBP) and glial fibrillary acidic protein (GFAP) we observed several features including astrocyte clasmatodendrosis with its endfeet damage, rupture of neurovascular units indicating BBB disruption, NF rupture along with diminished reaction to MBP [50]. Instead of this, we did not observe retraction bulbs within damaged axons suggesting the examined proteins deposition requires longer periods of survival time after TBI. This is in line with our current finding concerning cytoskeletal proteins where also we did not observe evident retraction bulbs in stainings performed. Nevertheless, despite the lack of retraction bulbs, the abovementioned findings are in this context significant as an early marker in the detection of the $T B I$ consequences in minutes. The study group presented a diminished KIF5B expression among oligodendroglia whereas in some cases we observed the increased expression in the vicinity and lumen of blood vessels in the white matter of frontal lobes with no reaction concerning red blood cells. As we previously reported, this is probably due to early phase activation and migration of macrophages/ phagocytic microglia cells towards blood vessels [51]. These phenomena are associated with clearing of cellular debris and initiation of neurorestorative processes or become dysregulated and act as a cytoand neurotoxic factor [42]. It was observed that microglia after lipopolysaccharide (LPS) stimulation become activated and convert its form to ameboid within several hours in vitro increasing the expression of cytoskeletal proteins, which is in line with our observations [1,61]. However, it is accepted that microglia react to TBI within minutes, what strengthens our remarks [17]. The observations in cytoskeletal stainings were also predictive of a neuronal/axonal injury even in cases where the head injury was not assumed to be direct the cause of death, which in some cases could remain undiagnosed during regular postmortem neuropathological examination and forensic autopsy similarly to what we stated previously [51]. The indicated axonal transport interruption also seems to be important in the context of primary non-lethal $\mathrm{TBI}$ victims who are clinically present with neurological symptoms like a headache, dizziness, chronic fatigue, deficits in attention, anxiety and many others [3,31]. In this case, visible changes in cytoskeletal protein expression indirectly give the evidence of occurred TBI followed by axonal transport interruption [24]. Collective microglia activation along with diminished reaction in oligodendroglia concerning the cytoskeletal proteins indicate their further and persisting dysfunction suggesting a link between TBI and subsequent neurodegenerative and demyelination diseases [23]. In our study context, the evident attention should be paid to Alzheimer's disease (AD) and its linked pathologies associated with proteinopathies along with other diseases such as Parkinson's disease (PD), amyotrophic lateral sclerosis (ALS) and dementia [22,69].

\section{Conclusions}

The study proved that dynein, dynactin, and kinesin staining should be considered a supplemental diagnostic tool for TBI in postmortem neuropathological examination and forensic autopsy. Furthermore, associated features in cytoskeletal stainings including neuronal body and axon reactive changes distribution along with the presence of various degree immunoreactivity in glial-like cells identified in the traumatic context should suggest the diag- 
nosis of TBI followed by TAl or at least the need of utilizing more specific techniques and markers. This additional staining procedure could be useful in the evaluation of lesions that could remain undiagnosed during a routine examination. In this regard, careful assessment and use of several methods, including timely autopsy and immunohistochemical staining for cytoskeletal proteins determination can contribute to more precise dating of the experienced severe and moderate TBI and aid more accurate identification of the TAl evolution.

\section{Acknowledgments}

This study was supported by internal grant $1 \mathrm{MB} /$ PM11/14 conducted in the years 2014-2015 from the Medical University of Warsaw. The project was implemented using the infrastructure financed by the European Union (EU) - the European Regional Development Fund (ERDF) within the "Infrastructure and Environment" operational program for years 2007-2013. No additional external funding and support was received for this study.

\section{Disclosure}

The authors report no conflict of interest.

\section{References}

1. Abd-el-Basset E, Fedoroff S. Effect of bacterial wall lipopolysaccharide (LPS) on morphology, motility, and cytoskeletal organization of microglia in cultures. J Neurosci Res 1995; 41: 222-237.

2. Adams JH, Doyle D, Ford I, Gennarelli TA, Graham DI, McLellan DR. Diffuse axonal injury in head injury: definition, diagnosis and grading. Histopathology 1989; 15: 49-59.

3. Alexander MP. Mild traumatic brain injury: pathophysiology, natural history, and clinical management. Neurology 1995; 45: 1253-1260.

4. Amunts K, Zilles K. Architectonic mapping of the human brain beyond brodmann. Neuron 2015; 88: 1086-1107.

5. Araque A, Navarrete M. Glial cells in neuronal network function. Philos Trans R Soc Lond B Biol Sci 2010; 365: 2375-2381.

6. Armstrong RC, Mierzwa AJ, Marion CM, Sullivan GM. White matter involvement after TBI: Clues to axon and myelin repair capacity. Exp Neurol 2016; 275: 328-333.

7. Blennow K, Hardy J, Zetterberg H. The neuropathology and neurobiology of traumatic brain injury. Neuron 2012; 76: 886-899.

8. Borgens RB, Liu-Snyder P. Understanding secondary injury. Q Rev Biol 2012; 87: 89-127.

9. Bramlett HM, Dietrich WD. Long-term consequences of traumatic brain injury: current status of potential mechanisms of injury and neurological outcomes. J Neurotrauma 2015; 32: 1834-1848.
10. Büki A, Okonkwo DO, Wang KK, Povlishock JT. Cytochrome $\mathrm{C}$ release and caspase activation in traumatic axonal injury. J Neurosci 2000; 20: 2825-2834.

11. Büki A, Povlishock JT. All roads lead to disconnection? Traumatic axonal injury revisited. Acta Neurochir (Wien) 2006; 148: 181-194.

12. Büki A, Siman R, Trojanowski JQ, Povlishock JT. The role of calpain-mediated spectrin proteolysis in traumatically induced axonal injury. J Neuropathol Exp Neurol 1999; 58: 365-375.

13. Cho C, Vale RD. The mechanism of dynein motility: insight from crystal structures of the motor domain. Biochim Biophys Acta 2012; 1823: 182-191.

14. Chodobski A, Zink BJ, Szmydynger-Chodobska J. Blood-brain barrier pathophysiology in traumatic brain injury. Transl Stroke Res 2011; 2: 492-516.

15. Christman CW, Grady MS, Walker SA, Holloway KL, Povlishock JT. Ultrastructural studies of diffuse axonal injury in humans. J Neurotrauma 1994; 11: 173-186.

16. Crooks DA. The pathological concept of diffuse axonal injury; its pathogenesis and the assessment of severity. J Pathol 1991; 165: 5-10.

17. Davalos D, Grutzendler J, Yang G, Kim JV, Zuo Y, Jung S, Littman DR, Dustin ML, Gan WB. ATP mediates rapid microglial response to local brain injury in vivo. Nat Neurosci 2005; 8: 752-758.

18. Duckworth JL, Grimes J, Ling GS. Pathophysiology of battlefield associated traumatic brain injury. Pathophysiology 2013; 20: 23-30.

19. Erb DE, Povlishock JT. Axonal damage in severe traumatic brain injury: an experimental study in cat. Acta Neuropathol 1988; 76: 347-358.

20. Eschbach J, Dupuis L. Cytoplasmic dynein in neurodegeneration. Pharmacol Ther 2011; 130: 348-363.

21. Faden Al, Loane DJ. Chronic neurodegeneration after traumatic brain injury: Alzheimer disease, chronic traumatic encephalopathy, or persistent neuroinflammation? Neurotherapeutics 2015; 12: 143-150.

22. Fleminger S, Oliver DL, Lovestone S, Rabe-Hesketh S, Giora A. Head injury as a risk factor for Alzheimer's disease: the evidence 10 years on; a partial replication. J Neurol Neurosurg Psychiatry 2003; 74: 857-862.

23. Gardner RC, Yaffe K. Epidemiology of mild traumatic brain injury and neurodegenerative disease. Mol Cell Neurosci 2015; 66: 75-80.

24. Geddes JF, Vowles GH, Beer TW, Ellison DW. The diagnosis of diffuse axonal injury: implications for forensic practice. Neuropathol Appl Neurobiol 1997; 23: 339-347.

25. Geddes JF, Whitwell HL, Graham DI. Traumatic axonal injury: practical issues for diagnosis in medicolegal cases. Neuropathol Appl Neurobiol 2000; 26: 105-116.

26. Gentleman SM, Nash MJ, Sweeting CJ, Graham DI, Roberts GW. Beta-amyloid precursor protein (beta APP) as a marker for axonal injury after head injury. Neurosci Lett 1993; 160: 139-144.

27. Giza CC, Hovda DA. The new neurometabolic cascade of concussion. Neurosurgery 2014; 75: S24-33.

28. Hammoud DA, Wasserman BA. Diffuse axonal injuries: pathophysiology and imaging. Neuroimaging Clin N Am 2002; 12: 205-216. 
29. Hånell A, Greer JE, McGinn MJ, Povlishock JT. Traumatic brain injury-induced axonal phenotypes react differently to treatment. Acta Neuropathol 2015; 129: 317-332.

30. Hawthorne C, Piper I. Monitoring of intracranial pressure in patients with traumatic brain injury. Front Neurol 2014; 5: 121.

31. Hemphill MA, Dauth S, Yu CJ, Dabiri BE, Parker KK. Traumatic brain injury and the neuronal microenvironment: a potential role for neuropathological mechanotransduction. Neuron 2015; 85: 1177-1192.

32. Hill CS, Coleman MP, Menon DK. Traumatic axonal injury: mechanisms and translational opportunities. Trends Neurosci 2016; 39: 311-324.

33. Hirokawa N, Noda Y, Tanaka Y, Niwa S. Kinesin superfamily motor proteins and intracellular transport. Nat Rev Mol Cell Biol 2009; 10: 682-696.

34. Hortobágyi T, Wise S, Hunt N, Cary N, Djurovic V, Fegan-Earl A, Shorrock K, Rouse D, Al-Sarraj S. Traumatic axonal damage in the brain can be detected using beta-APP immunohistochemistry within 35 min after head injury to human adults. Neuropathol Appl Neurobiol 2007; 33: 226-237.

35. Horton AC, Ehlers MD. Neuronal polarity and trafficking. Neuron 2003; 40: 277-295.

36. Jessen KR. Glial cells. Int J Biochem Cell Biol 2004; 36: 1861-1867.

37. Johnson VE, Stewart W, Smith DH. Axonal pathology in traumatic brain injury. Exp Neurol 2013; 246: 35-43.

38. Kan EM, Ling EA, Lu J. Microenvironment changes in mild traumatic brain injury. Brain Res Bull 2012; 87: 359-372.

39. Kardon JR, Vale RD. Regulators of the cytoplasmic dynein motor. Nat Rev Mol Cell Biol 2009; 10: 854-865.

40. Langlois JA, Rutland-Brown W, Wald MM. The epidemiology and impact of traumatic brain injury: a brief overview. J Head Trauma Rehabil 2006; 21: 375-378.

41. Li XY, Feng DF. Diffuse axonal injury: novel insights into detection and treatment. J Clin Neurosci 2009; 16: 614-619.

42. Loane DJ, Kumar A. Microglia in the TBI brain: The good, the bad, and the dysregulated. Exp Neurol 2016; 275: 316-327.

43. Ma M, Ferguson TA, Schoch KM, Li J, Qian Y, Shofer FS, Saatman KE, Neumar RW. Calpains mediate axonal cytoskeleton disintegration during Wallerian degeneration. Neurobiol Dis 2013; 56: 34-46.

44. Ma M. Role of calpains in the injury-induced dysfunction and degeneration of the mammalian axon. Neurobiol Dis 2013; 60 61-79.

45. Mallik R, Gross SP. Molecular motors: strategies to get along. Curr Biol 2004; 14: R971-982.

46. Meaney DF, Smith DH. Biomechanics of concussion. Clin Sports Med 2011; 30: 19-31.

47. Medana IM, Esiri MM. Axonal damage: a key predictor of out come in human CNS diseases. Brain 2003; 126: 515-530.

48. Miki H, Okada Y, Hirokawa N. Analysis of the kinesin superfamily: insights into structure and function. Trends Cell Biol 2005; 15: 467-476.

49. Ng HK, Mahaliyana RD, Poon WS. The pathological spectrum of diffuse axonal injury in blunt head trauma: assessment with axon and myelin strains. Clin Neurol Neurosurg 1994; 96: 24-31.

50. Olczak M, Kwiatkowska M, Niderla-Bielińska J, Chutorański D, Tarka S, Wierzba-Bobrowicz T. Brain-originated peptides as possible biochemical markers of traumatic brain injury in cerebrospinal fluid post-mortem examination. Folia Neuropathol 2018; 56: 97-103.

51. Olczak M, Niderla-Bielińska J, Kwiatkowska M, Samojłowicz D, Tarka S, Wierzba-Bobrowicz T. Tau protein (MAPT) as a possible biochemical marker of traumatic brain injury in postmortem examination. Forensic Sci Int 2017; 280: 1-7.

52. Roberts AJ, Kon T, Knight PJ, Sutoh K, Burgess SA. Functions and mechanics of dynein motor proteins. Nat Rev Mol Cell Biol 2013; 14: 713-726.

53. Roth S, Laan L, Dogterom M. Reconstitution of cortical Dynein function. Methods Enzymol 2014; 540: 205-230.

54. Rubiano AM, Carney N, Chesnut R, Puyana JC. Global neurotrauma research challenges and opportunities. Nature 2015; 527: S193-197.

55. Schweitzer JB, Park MR, Einhaus SL, Robertson JT. Ubiquitin marks the reactive swellings of diffuse axonal injury. Acta Neuropathol 1993; 85: 503-507.

56. Serbest G, Burkhardt MF, Siman R, Raghupathi R, Saatman KE. Temporal profiles of cytoskeletal protein loss following traumatic axonal injury in mice. Neurochem Res 2007; 32: 20062014.

57. Sherriff FE, Bridges LR, Sivaloganathan S. Early detection of axonal injury after human head trauma using immunocytochemistry for beta-amyloid precursor protein. Acta Neuropathol 1994; 87: 55-62.

58. Siedler DG, Chuah MI, Kirkcaldie MT, Vickers JC, King AE. Diffuse axonal injury in brain trauma: insights from alterations in neurofilaments. Front Cell Neurosci 2014; 8: 429.

59. Smith DH, Chen XH, Nonaka M, Trojanowski JQ, Lee VM, Saatman KE, Leoni MJ, Xu BN, Wolf JA, Meaney DF. Accumulation of amyloid beta and tau and the formation of neurofilament inclusions following diffuse brain injury in the pig. J Neuropathol Exp Neurol 1999; 58: 982-992.

60. Smith DH, Hicks R, Povlishock JT. Therapy development for diffuse axonal injury. J Neurotrauma 2013; 30: 307-323.

61. Stence N, Waite M, Dailey ME. Dynamics of microglial activation: a confocal time-lapse analysis in hippocampal slices. Glia 2001; 33: 256-266.

62. Stocchetti N, Carbonara M, Citerio G, Ercole A, Skrifvars MB, Smielewski P, Zoerle T, Menon DK. Severe traumatic brain injury: targeted management in the intensive care unit. Lancet Neurol 2017; 16: 452-464

63. Tang-Schomer MD, Johnson VE, Baas PW, Stewart W, Smith DH. Partial interruption of axonal transport due to microtubule breakage accounts for the formation of periodic varicosities after traumatic axonal injury. Exp Neurol 2012; 233: 364-372.

64. Tang-Schomer MD, Patel AR, Baas PW, Smith DH. Mechanical breaking of microtubules in axons during dynamic stretch injury underlies delayed elasticity, microtubule disassembly, and axon degeneration. FASEB J 2010; 24: 1401-1410.

65. Uchihara T. Silver diagnosis in neuropathology: principles, practice and revised interpretation. Acta Neuropathol 2007; 113: 483-499.

66. Uryu K, Chen XH, Martinez D, Browne KD, Johnson VE, Graham DI, Lee VM, Trojanowski JQ, Smith DH. Multiple proteins implicated 
in neurodegenerative diseases accumulate in axons after brain trauma in humans. Exp Neurol 2007; 208: 185-192.

67. Vaughan KT, Vallee RB. Cytoplasmic dynein binds dynactin through a direct interaction between the intermediate chains and p150Glued. J Cell Biol 1995; 131: 1507-1516.

68. Verweij BH, Muizelaar JP, Vinas FC, Peterson PL, Xiong Y, Lee CP. Impaired cerebral mitochondrial function after traumatic brain injury in humans. J Neurosurg 2000; 93: 815-820.

69. Washington PM, Villapol S, Burns MP. Polypathology and dementia after brain trauma: Does brain injury trigger distinct neurodegenerative diseases, or should they be classified together as traumatic encephalopathy? Exp Neurol 2016; 275: 381-388.

70. Weber JT. Altered calcium signaling following traumatic brain injury. Front Pharmacol 2012; 3: 60.

71. Xu J, Rasmussen IA, Lagopoulos J, Håberg A. Diffuse axonal injury in severe traumatic brain injury visualized using high-resolution diffusion tensor imaging. I Neurotrauma 2007; 24: 753 765.

72. Yaghmai A, Povlishock J. Traumatically induced reactive change as visualized through the use of monoclonal antibodies targeted to neurofilament subunits. J Neuropathol Exp Neurol 1992; 51: 158-176.

73. Zhao Z, Zhou Y, Tian Y, Li M, Dong Jf, Zhang J. Cellular microparticles and pathophysiology of traumatic brain injury. Protein Cell 2017; 8: 801-810 